\title{
Laser Projection Augmented Reality System for Computer Assisted Surgery
}

\author{
Neil Glossop ${ }^{1}$, Chris Wedlake ${ }^{2}$, John Moore $^{2}$, Terry Peters ${ }^{2,3}$, and Zhanhe Wang ${ }^{1}$ \\ ${ }^{1}$ Traxtal Technologies Inc, Toronto, Canada \\ ${ }^{2}$ University of Western Ontario \\ \{cwedlake, jmoore, tpeters\} eimaging.robarts.ca \\ ${ }^{3}$ Robarts Research Institute, London, Canada
}

\begin{abstract}
A new augmented reality apparatus was evaluated. The device uses scanned infrared and visible lasers to project computer generated information such as surgical plans, entry pints for probes etc, directly onto the patient. In addition to projecting the plan, the device can be integrated with a 3D camera and is capable of measuring the location of projected infrared laser spots. This can be used to ensure that the display is accurate, apply corrections to the projection path and to assist in registration. The projection system has its own Application Programmer's Interface (API) and is a stand-alone add-on unit to any host computer system. Tests were conducted to evaluate the accuracy and repeatability of the system. We compared the locations of points projected on a flat surface with the measurements obtained from a tracked probe. The surface was rotated through 60 degrees in 5 degree increments and locations measured from the two devices agreed to within $2 \mathrm{~mm}$. An initial host application was also developed to demonstrate the new unit. Fiducials representing vertices along a proposed craniotomy were embedded into a plastic skull and a projection path defining the craniotomy was calculated. A feedback-based optimization of the plan was performed by comparing the measurement taken by the camera of these coordinates. The optimized plan was projected onto the skull. On average, the projection deviated by approximately $1 \mathrm{~mm}$ from the plan. Applications include identification of critical anatomical structures, visualization of preplanned paths and targets, and telesurgery or teleconsultation.
\end{abstract}

\section{Introduction}

Image Guided Surgery systems traditionally display intraoperative navigation information on a computer display placed in the operating room. The navigation information is derived from preoperative or intraoperatively acquired images which are registered with the actual patient. Typically, a 3D camera is used to track surgical instruments, and representation of the position and orientation of the instruments are calculated and superimposed on the preoperative data. Sometimes these displays take the form of complex three-dimensional renderings of the anatomy or reformats of the two-dimensional data in the vicinity of the tip of the instrument. 
Augmented Reality (AR) systems are designed to superimpose this type of information over the surgeon's view of the operative site, enabling him to better concentrate on the intervention. The use of these systems is analogous to the concept of the helmet-mounted or heads-up display commonly used in aircraft cockpits to display instrument readings. These displays reduce the amount of visual re-focusing and head re-positioning, caused by the motion going from screen to patient to screen. Mental registration and transformation of the images to the patient is also reduced or eliminated, which is often perceived as a significant advantage.

Most previous work with AR in surgical environments has concentrated on the use of virtual reality goggles, operative microscopes, and semi-transparent mirrors. For example, several research and commercial systems, are now available that inject computer generated images directly into the optical path of microscopes [1] or surgical loupes [2]. Preplanned targets such as tumors or biopsy trajectories are segmented or planned prior to surgery and then adjusted for the current location of the viewing optics and focal plane and then displayed with the live optical stream.

Image overlay methods employ a partially reflecting mirror placed between the surgical site and the surgeon and include the system developed by Blackwell et al. [3] and the Dextroscope ${ }^{\mathrm{TM}}$ from Volume Interactions. Part of the view comes from a partial reflection of a (usually) stereoscopic computer display, and the other part comes from a direct view of the patient through the glass. In these systems, the surgeon's head is tracked to make allowances for perspective. A new system also using a semi-transparent mirror approach was recently described by Liao et al. [4]. A real 3D image (Integral Videography) is projected into the patient volume. Here, the full 3D image is perceived to be in the correct place independent of viewer position.

Recently, projection techniques have been demonstrated. Hoppe et al. [5] used a video projector to display a surgical plan on the patient by using the patient as a screen. Hoppe was further able to obtain surface morphology information by using structured light ("light-striping") techniques and two video cameras. This syetem is large however, and requires a special self-contained cooling system to minimize disturbance of the laminar airflow in the operating room. The optical tracking system in this device was custom designed to also facilitate the structured light measurements, and has only limited ability to track surgical instruments.

Sugano et al. [6] used scanned lasers attached to either side of a camera system to guide total hip arthroplasty. Laser beams were scanned in planes, and the intersection line of the two planes was used to guide the trajectory and entry point of surgical instruments. The system was not used to display complex patterns with the lasers.

The work presented in this paper illustrates the use of a new method of projection based augmented reality. By rapidly scanning laser beams in two axes, it is possible to project surgical plans onto a patient directly without the need for interposing optics between the eye and the patient. The projected contours can be used to guide the surgeon to carry out a plan and avoid critical structures. The device also enables the measurement of the surface morphology directly, using the same 3D digitizing camera system currently used in most IGS workstations as an instrument tracking system. This is done without impeding or altering the basic functionality of the camera in its capacity to track instruments. 


\section{Materials and Methods}

\subsection{XarTrax System}

The XarTrax (Traxtal Technologies, Toronto Canada) is an augmented reality and measurement system using lasers to project spots of light on the patient. Because "persistence of vision" effects cause rapidly scanned laser spots to appear as lines, it is possible to project complex patterns by quickly moving the spots according to recorded patterns. At the time of writing, this was the first use of the prototype system outside of the manufacturer's facility.

The XarTrax consists of a control unit and a projector. The projector houses visible and infrared (IR) lasers that can be steered by two perpendicular galvanometrically controlled mirrors. The laser beams are aligned coaxially so they project overlapping spots on surfaces placed in front of the projector. These beams can be selectively turned on and off so that either the visible or IR beam or both are powered at any one time.

The control unit contains amplifiers, power supplies and embedded controllers required to operate the optical projector. It accepts commands from a host computer delivered over a serial interface. The system is designed so that the projector can be mounted rigidly in conjunction with infrared positioning cameras such as the POLARIS (NDI, Waterloo, Canada), which is capable of "reading" the $(\mathrm{x}, \mathrm{y}, \mathrm{z})$ location of an infrared laser spot projected onto a surface.

\subsection{Controlling the System}

The software interface of the XarTrax consists of a built-in Application Program Interface (API) that is a superset of the API of the POLARIS camera. API commands are sent from the host computer running the application software through a serial port. The controller either executes the command or forwards it to the camera via an output serial port. The API has been constructed so that camera-only software can usually run unaltered even when the XarTrax is inserted into the system. Compared to using the POLARIS camera alone, there is currently up to a $15 \mathrm{~ms}$ lag in each direction caused by the overhead of the device to retransmit the commands and responses over the serial line.

There are two different ways of using the XarTrax. "Direct Mode" is employed for immediate control of the system. Here, the host sends a pair of numbers to the system, which represent the angles through which the mirrors must be rotated to direct the beam to the appropriate target. Likewise, the laser states can be changed by instructing the visible or infrared laser to turn on or off. Because commands are issued sequentially, it is possible to mix POLARIS commands with XarTrax commands using Direct Mode. This allows both the sampling of the IR laser position and tracking of tools.

In "Arbitrary Waveform Mode", a complete table of data, consisting of angular positions of the mirrors as well as the state of the lasers in each position, is loaded into the XarTrax display buffer. A command is issued to control the number of times 
that the data table should be displayed along with the speed of the display. Finally a "start" command is generated to begin the playback.

Typically the speeds attained using Arbitrary Waveform Mode are much greater than those attainable using the Direct Mode, since the data are transferred to the projector in a single block rather than continuously. The projection is handled by the controller computer enabling a host application to continue without looking after the details of the projection.

\subsection{Experiments}

We performed some initial accuracy studies in addition to developing a general-purpose application to evaluate the general use of the system.

The first test evaluated the accuracy of measuring the projected beam location using the camera. The surface was rotated to determine the effect of angle. The XarTrax was first mounted to a Polaris camera as shown in Fig 1., with the camera's z-axis parallel to the XarTrax lasers. A flat aluminum plate was used as a target for the beam and arranged using a

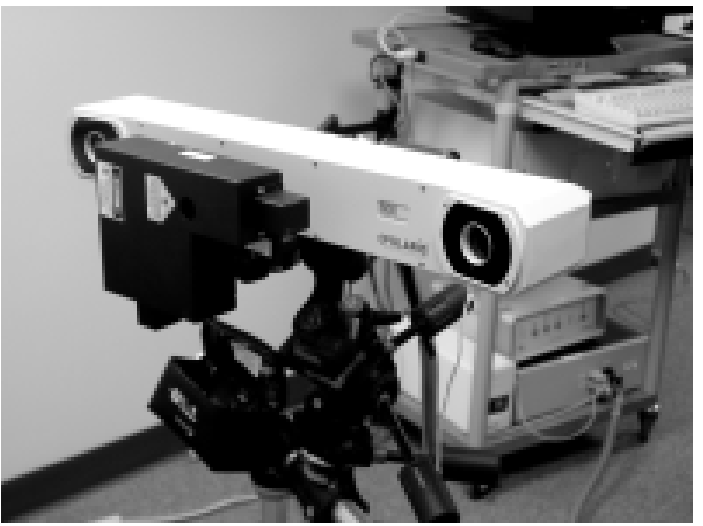

Fig. 1. The XarTrax device mounted upon a POLARIS optical tracking system.

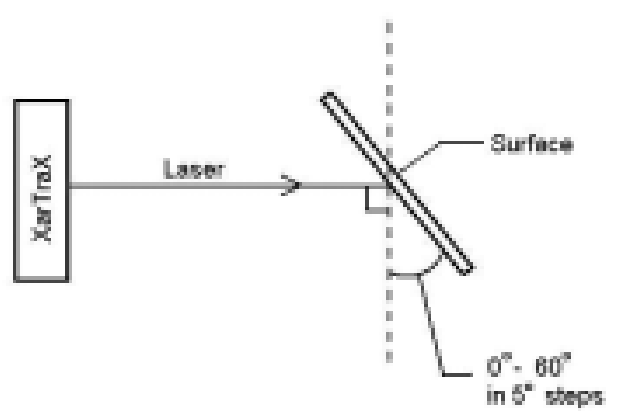

Fig. 2. Experimental configuration to assess the performance of XarTrax as a function of the angle between the beam and the surface. for the surface was 60 degrees (Fig 2.). At 60 degrees the Polaris began picking up two laser points, due to a reflection. Only a single "passive" IR target (i.e. the laser spot) was visible to the camera, so this was not a "stray" marker. 
Strays appear when multiple passive targets are coplanar to the POLARIS detectors. The test was run twice, generating 20 data sets for each angle, at a distance of $1600 \mathrm{~mm}$ between the plane and the XarTrax equipment. The passive probe was also sampled independently by placing it 20 times on a stationary laser spot to determine the inherent error in the placement process that might be expected in a clinical setting.

A second experiment measured the repeatability of projection and measurement. A flat wood plate, $60 \mathrm{~cm}$ high by $30 \mathrm{~cm}$ long that was fixed to a rigid metal base. Six laser point locations forming a $3 \times 2$ grid were sequentially projected and measured on the surface by changing the $\mathrm{x}$ and $\mathrm{y}$ angles. The POLARIS was placed at distances of $1750 \mathrm{~mm}$ and $1900 \mathrm{~mm}$ and $2100 \mathrm{~mm}$ from the surface. The displacement between the six points was varied between 2 and $50 \mathrm{~mm}$.

Finally, an application was developed to demonstrate the system in a typical surgical setting by simulating the projection of a craniotomy. Fiducials representing vertices along a closed projection path were embedded into a plastic skull. A CT scan was made of the skull, and a 3D model of the skull and fiducials was reconstructed using the Visualization Toolkit (VTK) A craniotomy projection path was then created using the model, consisting of the lines joining the fiducials. This allowed us to verify accuracy by comparing the projection with the fiducials. Both the coordinates of each vertex in image space, and the normal vector were recorded, along with an average normal vector of the contour. We also determined the image space coordinates of seven additional registration fiducials placed around the skull.

The plan and image space registration points were transferred to a host application that controlled the projector and camera. The plastic skull was attached to a Mayfield clamp (OMI, Cincinnati Ohio) and an active reference (LED based) tracker (Traxtal) was attached using a support arm and pointed toward the camera.

A paired-point registration was performed, using a probe to touch the registration fiducials points. The resulting registration matrix related the image space coordinates to the camera space coordinates. A rough transformation between the projector coordinate system and the camera coordinate system was calculated using previous measurements, enabling us to determine the angle between the projector's origin and the average contour normal once the registration was performed. The cameraprojector assembly was then manually moved and tilted to place the projector as normal to the surface as possible. A simple targeting user interface was employed for this which allowed us to manually position the projector in about 10 seconds.

A calculation was performed to determine the mirror angles required to project the preplanned pattern. While it is possible to project the beam immediately, we implemented different optimization schemes to attempt to determine and even correct the beam positions to match the a priori geometry.

The infrared beam was steered to each point along the path in turn. A feedbackbased optimization of the plan was performed by comparing the measurement taken by the Polaris camera of these coordinates with the preplanned projection coordinates according to the 3D model. The projection location of the point was slightly changed and the new error determined and the process repeated until the error had been minimized. The optimized plan was then projected onto the patient with the visible beam. Optimization was also performed using a single point instead of every point.

\footnotetext{
${ }^{1}$ www.kitware.com
} 
This optimization step also has the effect of refining the transformation between camera and projector coordinate spaces.

We measured the projection error manually using a caliper to determine the deviation of the visible projection beam from the fiducials that made up the vertices of the plan. It was possible to compensate for motion of the skull during projection using the dynamic reference. Typically we projected the craniotomy path twenty times consecutively, at a scan rate of 2000 points per second. This was followed by sampling the position of the reference frame, and correction of the projection location if necessary. The corrected scan was then re-played and the sequence repeated.

\section{Results}

We were able to detect and measure the laser spot over the range from 0 to 60 degrees before reflections started to cause difficulties. Rapidly repeated measurements of the laser spot were inherently noisier than the rapid samples obtained from the stationary probe. The average of the single point sampling standard deviation 13 points with 10 samples each (total 130 points) was $1.2 \mathrm{~mm}$ with the laser compared to $0.13 \mathrm{~mm}$ with a tracked probe. The magnitude of the distance between the probe and laser averaged $2.0 \mathrm{~mm}$ over all the angles, ranging from $0.74 \mathrm{~mm}$ to $3.3 \mathrm{~mm}$. While the measured distances were lowest for 0 degrees (i.e. perpendicular to the surface), and highest at 60 degrees there was no clear systematic relationship with angle. It is also impossible to tell from this experiment if the probe or the laser measurement was in error.

Testing with the probe alone showed that simply removing and replacing the probe to the same laser spot causes an average variation of $0.9 \mathrm{~mm}$ at a distance of $1700 \mathrm{~mm}$ from the camera. Additional errors that can contribute to the difference in measured position can arise from small misalignments of the visible and infrared lasers and from error in the absolute probe position, which relies on pivoting to determine offset.

When sampling the probe, location data from each of the three reflective spheres will contribute to the rigid body calculation from which the probe tip is calculated, increasing its accuracy. Because of this, we expect that single point sampling will generally always produce results that are not as accurate as can be obtained when extrapolating a single point from three points that were used to calculate the probe tip position. The results of all tests indicated that it is necessary to average the laser measurements between 10 and 30 times to obtain the best readings.

These initial studies show that the system is robust and the repeatability is high. The repeatability study showed that at a XarTrax-to-target distance of $1750 \mathrm{~mm}$, the distance between subsequent measurements of the same six points yielded a combined projection-measurement distance variation between $0.3 \mathrm{~mm}$ and $1.1 \mathrm{~mm}$ with a mean difference of $0.66 \mathrm{~mm}$. This discrepancy increased to an average of $1.0 \mathrm{~mm}$ at 1900 $\mathrm{mm}$, and $1.24 \mathrm{~mm}$ at $2100 \mathrm{~mm}$.

Accuracy under simulated clinical conditions was approximately $1 \mathrm{~mm}$ in this preliminary study. Errors in the system consist of a superposition of errors from the plan (i.e the CT scan and the selection of coordinates), the registration, and projector itself. We also note that a path on the target is projected most accurately when the beam is approximately normal to the surface of the target object. 
The overall operational paradigm that was used worked well. This involved preplanning using the "pre-operative" image data, followed by registration and optimization of the path prior to projection. The use of the dual lasers has many advantages, enabling verification that the projected point will fall on the desired location on the target prior to projection. This adds an extra element of safety and can potentially increase the accuracy of the system. The system is also capable of performing point-cloud based registration or other digitization with the infrared laser. The infrared laser is also visible to many standard low-cost CCD cameras, so measurements and trial projections can be performed without specifically involving the operating-room team. Because of its compatibility with the POLARIS API, the device was easy to integrate into conventional IGS applications, requiring no special hardware or software changes.
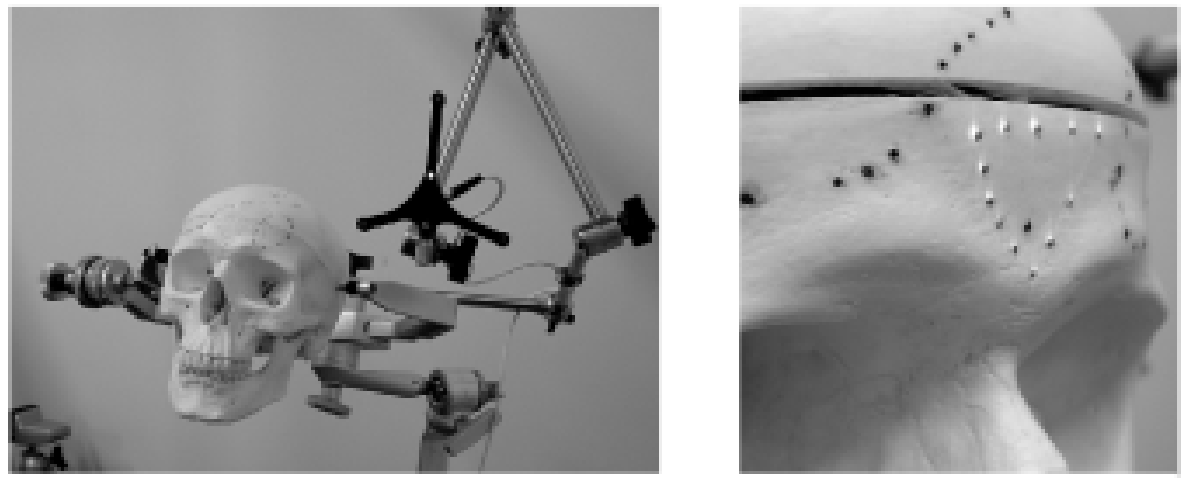

Fig. 3. Experimental setup for clinical study. The photo at right shows the projected pattern overlaying the target fiducials used to create the plan.

The XarTrax system is more limited in the amount and type of information that is displayed than conventional augmented reality systems that inject video images. The system is monochromatic with a limited update rate due to the rotational inertia of the mirrors. This enables it to display vector objects such as lines, paths and points most efficiently, rather than full raster or texture mapped images. This is not a major limitation, since critical information can often be displayed most effectively and with least amount of distraction in a simple form. Laser projection can also be made to display extremely bright and thin, well defined lines. Video projectors are limited by pixel size and tend to project less vivid images than lasers are capable of.

\section{Conclusion}

A dual wavelength system that can be used to project the positions of planned surgical paths or entry points directly onto a patient has been evaluated. Because the device can be used to measure the positions prior to actually projecting the information, the path can be verified and corrected if necessary. 
Positional sampling with the XarTrax laser and the POLARIS produces results that were noisier and slightly less accurate than with a probe. This is a limitation resulting from the use a single sampling point rather than the three or more on typical probes. The accuracy is sufficient for many image guided surgery applications however.

Possible applications include identification of critical anatomical structures, visualization of preplanned paths and targets, and telesurgery as an alternative to robotic systems. The overall clinical accuracy of the device was measured at approximately $1 \mathrm{~mm}$.

Acknowledgements. We wish to thank Chris Tsui of Traxtal for the VTK modeling. D. Lindisch and K. Cleary of the ISIS center, Georgetown University Medical Center provided the CT scans used in this project. We also wish to thank Ravi Gupta of the Robarts Institute for helping to coordinate this study.

\section{References}

1. Edwards, P. J., King, A. P., Maurer, C. R., de Cunha, D. A., Hawkes, D. J., Hill, D. L. G., Gaston, R. P., Fenlon, M. R., Jusczyzck, A., Strong, A. J., Chandler, C. L., Gleeson, M. J. Design and Evaluation of a System for Microscope-Assisted Guided Interventions (MAGI). IEEE Trans Med Imaging;19(11):1082-9. 2000

2. Birkfellner, W., Figl, M., Huber, K., Hummel, J., Hanel, R., Homolka., P., Watzinger, F., Wanschitz, F., Ewers, R., and Bergmann, H. Calibration of Projection Parameters in a Head-Mounted Display for Augmented Reality Visualization in Image Guided Therapy. Proc. SPIE Medical Imaging 2001, San Diego CA 4320, 17-23 Feb. 2001.

3. Blackwell, M., Nikou, C., DiGioia, A. M., Kanade, T. An Image Overlay System for Medical Data Visualization. Med Image Anal. 4(1):67-72. 2000.

4. Liao, H, Hata N, Iwaraha M, Nakajima S, Sakuma I, Dohi T.. High resolution stereoscopic surgical display using parallel integral videography and multi-projector. Proc MICCAI 2002, T Dohi and R Kikinis (eds), LNCS 2489, pp65-92, 2002.

5. Hoppe, H., Kuebler, C., Raczkowsky, J., Woern, H. and Hassfeld, S. A Clinical Prototype System for Projector-Based Augmented Reality: Calibration and Projection Methods. Proc. 16th Int. Congress and Exhibiton on Computer Assisted Radiology and Surgery (CARS) 2002, Springer, p. 1079, H. U. Lemke, M. W. Vannier, K. Inamura, A. G. Farman, K. Doi, and J. H. C. Reiber Ed, 2002.

6. Sugano, N., Sasama, T., Nishihara, S., Nakase, S., Nishii, T., Miki, H., Momoi, Y., Yoshinobu, S., Nakajima, Y., Tamura, S., Yonenobu, K., Ochi, T. Clinical Applications of a Laser Guidance System with Dual Laser Beam Rays as Augmented Reality of Surgical Navigation. Proc. 16th Int. Congress and Exhibition on Computer Assisted Radiology and Surgery (CARS) 2002, Springer, p. 281, H. U. Lemke, M. W. Vannier, K. Inamura, A. G. Farman, K. Doi, and J. H. C. Reiber Ed, 2002. 